\title{
Texture Analysis in the Evaluation of Covid-19 Pneumonia in Chest X-Ray Images: a Proof of Concept Study
}

\section{Armando Ugo Cavallo ( $\sim$ armandocavallo90@gmail.com )}

Department of Biomedicine and Prevention, University of Rome "Tor Vergata", Rome, Italy

\section{Jacopo Troisi}

Theoreo srl - Spin-off company of the University of Salerno, Italy https://orcid.org/0000-0003-2962-7379

\section{Marco Forcina}

Division of Radiology, San Carlo di Nancy Hospital, GVM Care and Research, Rome, Italy

\section{Piervalerio Mari}

Division of Internal Medicine, San Carlo di Nancy Hospital, GVM Care and Research, Rome, Italy

\section{Valerio Forte}

Division of Radiology, San Carlo di Nancy Hospital, GVM Care and Research, Rome, Italy

\section{Massimiliano Sperandio}

Division of Radiology, San Carlo di Nancy Hospital, GVM Care and Research, Rome, Italy

\section{Sergio Pagano}

Department of Physics “E.R. Caianello”, University of Salerno, Salerno, Italy

\section{Pierpaolo Cavallo}

Department of Physics “E.R. Caianello”, University of Salerno, Salerno, Italy

\section{Roberto Floris}

Neuroradiology Unit, Department of Biomedicine and Prevention, University of Rome "Tor Vergata”, Rome, Italy

Francesco Garaci

Neuroradiology Unit, Department of Biomedicine and Prevention, University of Rome "Tor Vergata”, Rome, Italy

\section{Research Article}

Keywords: Radiomics, Texture Analysis, COVID-19, Chest X-Ray

Posted Date: June 25th, 2020

DOI: https://doi.org/10.21203/rs.3.rs-37657/v1

License: (c) (1) This work is licensed under a Creative Commons Attribution 4.0 International License. Read Full License

Version of Record: A version of this preprint was published at Current Medical Imaging Formerly Current Medical Imaging Reviews on January 12th, 2021. See the published version at https://doi.org/10.2174/1573405617999210112195450. 


\section{Abstract}

Objectives: One of the most challenging aspects related to Covid-19 is to establish the presence of infection in early phase of the disease. Recently Artificial Intelligence systems for the diagnosis of Covid-19 related pneumonia on Chest $X$ ray (CXR) or chest CT have been tested with variable, but not negligible, accuracy. Texture analysis might be an additional tool for the evaluation of CXR in patients with clinical suspicion of Covid-19 related pneumonia.

Methods: CXR images were accessed from a publicly available repository (https://www.kaggle.com/tawsifurrahman/covid19-radiography-database). Lung areas were manually segmented using a polygonal regions of interest (ROI) covering both lung areas, using MaZda, a freely available software for texture analysis. A total of 308 features per ROI was extracted. One hundred-ten Covid-19 CXR images were selected for the final analysis

Results: Six models, namely NB, GLM, DL, GBT, ANN and PLS-DA were selected and ensembled. According to Youden's index, the Covid-19 Ensemble Machine Learning (EML)-Score showing the highest AUCROC $(0.971 \pm 0.015)$ was 132.57. Assuming this cut-off the EML model performance was estimated evaluating both true and false positive/negative, resulting in $91.8 \%$ accuracy with $93 \%$ sensitivity and $90 \%$ specificity. Moving the cut-off value to -100 , although the accuracy resulted lower (90.6\%), the EML showed $100 \%$ sensitivity, with $80 \%$ specificity

Conclusion: Texture analysis of CXR images and machine learning algorithms may help in differentiating patients with Covid-19 pneumonia. Despite several limitations, this study can lay ground for future researches in this field and help developing more rapid and accurate screening tools for these patients.

\section{Key Point}

Texture analysis of Chest X-Ray images and machine learning algorithms may help in differentiating patients with Covid-19 pneumonia from patient with other pneumonia with a different etiology.

\section{Introduction}

Since December 2019, a series of unidentified interstitial pneumonias were reported in the city of Wuhan in the Hubei province, China [1]. Investigations have been conducted by the Chinese Centre for Disease Control and Prevention and a novel coronavirus from human epithelial cells have been isolated, defined as the causative agent of the outbreak and named severe acute respiratory syndrome coronavirus 2 (SARS-CoV-2) [2]. Nowadays, the disease caused by the SARS-CoV-2 (Covid-19) is officially pandemic and worldwide spread [3].

To date, the real-time reverse-transcriptase-polymerase-chain-reaction (RT-PCR) of nasopharyngeal swabs has been used in order to confirm the diagnosis of Covid-19 in patients when respiratory symptoms such as cough, fever or dyspnea on exertion as reported in [4, 5]. The detection of the SARS-CoV-2 have been investigated in different types of clinical specimens such as bronchoalveolar lavage (BAL), sputum, feces and blood revealing that extrapulmonary transmission is possible and suggests a systemic involvement of the infection [6]. Also, the detection of antibodies anti SARS-CoV-2 proteins depends on the host immune response and is important for patients with mild Covid-19, that may be diagnosed after the first two or three weeks since the disease onset [7]. Covid-19 diagnosis based on chest imaging showed an higher sensitivity similarity to nasopharyngeal swabs one, with a quick results production 
[8]. In this scenario, radiological examinations are pivotal for the clinicians, allowing to early diagnose and evaluate the progression of Covid-19 in the emergency frontline.

Chest computed tomography (CT) demonstrate a high sensitivity in diagnosing Covid-19, showing a low rate of missed diagnosis [8] and providing clinicians with a valuable tool when nasopharyngeal swab RT-PCR results are notconclusive or unavailable. However, remarkable limitations have been reported for chest CT diagnosis of Covid-19. SARS-CoV-2 disease could be misdiagnosed because the radiological features are non-specific for the novel coronavirus and a typical pattern had not been found to date [9]. In fact, the CT findings of Covid-19 may overlap with those associated either with viruses from a different family such as adenovirus or either with the same cluster such as the MERS-CoV and the SARS-CoV [10]. Moreover, the use of the chest CT as a first-line investigation places a significant burden on radiology department and the infection control may be challenging [11].

Although containment measures decreased the total number of Covid-19 reported during the current pandemic, countries such as Italy, Spain, the United States and the United Kingdom have been particularly affected by SARSCoV-2 and their national health systems were overwhelmed [12]. In such demanding outline, the chest X-ray (CXR) can be considered a fast, easy and available screening tool for clinicians with a good baseline sensitivity of 69\% [11]. The bilateral involvement, the peripheral and lower zone dominance are common chest CT findings, but they can also be recognized in CXR, though no specific chest imaging signatures have been defined by expert radiologists in order to rule out non-Covid-19 pneumonia.

Radiomics, which is the quantitative extraction and analysis of data from medical images [13, 14], is a promising tool to analyze and categorize medical images and has been applied in CT and Magnetic Resonance Imaging (MRI) images for the evaluation of different diseases $[15,16]$. The radiomic investigation applied to the CT can provide clinicians with quantitative analysis suggesting that radiomic signature is a potential, useful tool for the Covid-19 diagnosis [17].

Recently Artificial Intelligence systems for the diagnosis of Covid-19 related pneumonia on CXR or chest CT have been tested with variable but not negligible accuracy [18-20].

Texture analysis might be an additional tool for the evaluation of CXR in patients with clinical suspicion of Covid-19 related pneumonia.

In this study, we investigate the CXR radiomics of patients affected by Covid-19 to find a specific radiomic signature that characterizes Covid-19 and allows clinicians to discriminate non-Covid-19 pneumonias using CXR as a screening tool. Radiomic analysis of CXR could be a useful tool in Covid-19 diagnosis and may provide pivotal advantages for clinicians at the frontline, such as a fast investigation while waiting for the nasopharyngeal swab RTPCR result. Moreover, it gives the possibility to use portable CXR device to avoid infections in the emergency department or in screening campaigns, reducing the burden on CT units.

\section{Materials And Methods}

\section{Image analysis}

CXR images were accessed from a publicly available repository (https://www.kaggle.com/tawsifurrahman/covid19radiography-database [21]. Images were downloaded on May $8^{\text {th }}, 2020$ and were analyzed using MaZda 4.6 (Institute of Electronics, Technical University of Lodz, Lodz, Poland) [22], a segmentation and texture analysis software. 
Lung areas were segmented using a polygonal regions of interest (ROI) covering both lung areas by two radiologists in consensus. Care was taken in not including mediastinal or abdominal structures in the ROI (Figure 1 A-B)..

Gray-level normalization was performed by rescaling the histogram data to fit within $\mu$-gray-level mean $\pm 3 \sigma$ ( $\sigma$-graylevel standard deviation) to reduce contrast and brightness variations that might impair texture feature quantification [23].

A total of 308 features per ROI were extracted. Texture features were calculated from the gray level histogram analysis, co-occurrence matrix calculated in 5 distances, run-length matrix calculated in 4 directions, autoregressive model and Wavelet transform.

The detailed description of the radiomic features is freely available on the online MaZda 4.6 manual.

One hundred-ten non Covid-19 related interstitial pneumonia CXR out of 1345 enclosed in the repository were randomly selected for the final analysis (Figure $1 C$-D), using the same exclusion criteria of Covid-19 images.

One hundred-ten Covid-19 CXR images out of 219 enclosed in the repository were selected for the final analysis. Exclusion criteria were (Figure 1 E-H):: a) presence of wires, ECG electrodes, Pacemaker, Central Venous Catheter on lung areas $(n=45), b)$ presence of annotations $(n=7), c)$ very poor image quality $(n=16)$, d) latero-lateral chest projection $(n=12)$, e) doubled image in the repository $(n=29)$.

\section{Statistical analysis}

After samples separation into training $(n=132)$ and test $(n=88)$ sets $(60: 40$ ratio), the training test was used to train 11 classification models: Partial Least Square Discriminant Analysis (PLS-DA), Naïve Bayes (NB), Generalized Linear Model (GLM), Logistic Regression (LR), Fast Large Margin (FML), Deep Learning (DL), Decision Tree (DT), Random Forest (RF), Gradient Boosted Trees (GBT), artificial Neural Network (aNN) and Support Vector Machine (SVM). Fine hyperparameter tuning was implemented to find the best combination, maximizing the models' accuracy, avoiding overfitting (evaluated by means of a cross-validation procedure on the training dataset). At the same time, models were trained using a large number of features subsets.

Features were first screened to be included in the model using 3 criteria: a) correlation (features that too closely, or not at all, mirror the Yes/No diagnosis criterion), b) stability (features where nearly all values are identical), c) missing (features with missing values) and then mixed to find the combination which would maximize the model's accuracy avoiding overfitting.

Models showing an accuracy higher of $80 \%$ were selected and ensembled using a voting scheme assuming both the cross-validation accuracy and the confidence (i.e. distance from classification margin) as a vote weight. Ensembling was performed according to Troisi et al. [24-26]. Briefly, for the images classified as "Covid-19", the scores (the products of model accuracy and classification confidences) were left untouched, while for each "Other" classification the scores were multiplied by -1 . Finally, a Covid-Ensemble Machine Learning (EML)-score was calculated for each sample by summing all the individual classification model scores.

The area under receiver operating characteristic (AUCROC) curve, as well as sensitivity, specificity, positive and negative predictive values, positive and negative likelihood ratios and accuracy were calculated to evaluate the ability of the Covid-EML-score to predict the SARS-CoV-2 pneumonia etiology. A cut-off point was evaluated as the score 
maximizing the Youden's Index (sensitivity + specificity - 1). DeLong et al. [27] non-parametric approach was used to compare the AUCROC. Moreover, cut-off maximizing sensitivity was also considered.

The overall diagnostic performance of the proposed score was investigated using a confusion matrix to summarize the results obtained using samples in the test set.

Statistical analyses were performed by means of Rapid Miner Studio ver. 9.7.0 (RapidMiner GmbH, Boston, MA, USA) and R-Studio ver. 1.2.5042 [28].

\section{Results}

Globally, 74,651 models were built and tested, to find the best combination of hyperparameters and features subset able to maximize the diagnostic accuracy of the investigated models. Six models, namely NB, GLM, DL, GBT, ANN and PLS-DA showing an accuracy $>80 \%$ with no overfitting were selected and ensembled. The models performances are reported in table 1. DL and GBT models show the best sensitivity (100\%), while aNN shows the highest specificity (98\%). According to Youden's index, the Covid-EML-Score showing the highest AUCROC $(0.971 \pm 0.015)$ is 132.57. According to this cut-off the EML model performance is estimated evaluating both true and false positive/negative, resulting in $91.8 \%$ accuracy with $93 \%$ sensitivity and $90 \%$ specificity. Moving the cut-off value to -100 , although the accuracy resulted lower (90.6\%), the EML shows 100\% sensitivity, with $80 \%$ specificity (see table 1 and figure 2).

A graphical representation of classes (Covid-19 Vs Other) separation achieved by means of the PLS-DA model is provided in figure 3A and supplementary S1. A volcano plot representation of all features is also reported (figure 3B): 22 features show both a $p$-value $<0.05$ and a fold change higher of 1 , while no feature showed both a fold change $<-1$ and a $\mathrm{p}$-value $<0.05$.

In figure $4 \mathrm{~A}$ an upset representation of the relevant features (with a weight $>0$ ) for each model is shown. The 10 features selected (used in at least 4 models with a weight larger than zero) are also showed in figure 4B according to their normalized mean values. The Euclidean distance of these normalized means trends, allows the clustering of these features in two separate groups: one, including S1_1Contrast, S3_0Contrast, S3_0DifVarnc, S3_3Contrast, S0_5DifVarnc, WavEnHLs-1 and WavEnHHs-5, that are higher in Other group and one, including S1_1AngScMom, WavEnLLs-5 and WavEnLLs-6, showing higher values in Covid-19 group. All the features weight are also reported in Supplementary S2.

\section{Discussion}

Radiomics is a rapidly evolving field which assumes that textural features of medical images may reflect underlying microstructural features associated to specific disease. Artificial intelligence (Al) may improve the quantification and the diagnostic accuracy of radiomic features. Thus, analysis of radiomic features with Al systems has the potential to improve patient stratification, treatment planning, and therapy monitoring, and may be combined with clinical and genomic data.

In this scenario, well balanced and high accuracy texture analysis algorithms may speed up the clinical work up of patients with suspected SARS-CoV-2 infection.

To date, Al diagnostic systems for Covid-19 interstitial lung disease have been implemented in CT and chest X-ray images with variable but not negligible accuracy [18-20]. These approaches show a good diagnostic accuracy but do not consider textural features of images. A combined approach of Al and radiomic feature extraction and analysis

Page 5/12 
may help in rapidly identifying Covid-19 patients and, additionally, might help in understanding textural and histological features of Covid-19 related interstitial pneumonia.

RT-PCR is the gold standard [29] for diagnosis of Covid-19, even if a high false negative rate has been reported. The presence of viral pneumonia is one of the most important features of this infection, and imaging techniques play a role in its diagnostics and management, as they already do for all the viral pneumonia types. The most widespread imaging used for thorax are the CXR, followed by CT, which is more complex to perform in patients with limited mobility, e.g. those in Intensive Care Units (ICU) or those who are isolated at home, and Lung Ultrasonography (LUS), not yet widespread and operator dependent.

From a clinical practice point of view, CXR is easier to perform in ICU and/or at home, during isolation in disease or after discharge, and its diagnostic parameters are very well established and standardized, while LUS [30] is also easy to do at the bedside and/or at home, but it is still quite operator dependent and needs standardized protocols [31]. Indeed, speaking in terms of "quick response rate", as rapid progression is a main risk, imaging techniques can be of great advantage to have a constant possibility of bedside monitoring, especially if the use of an automated texture analysis may greatly improve diagnostic usefulness of CXR, giving to the clinician, who can still keep making a qualitative interpretation of the images, an added value of objective, big data based, analysis.

From the availability point of view, CXR, together with LUS, appear to be the most readily available technologies, as the CT needs fixed facilities and patient movement, while PCR needs time and, due to possible local peaks of first diagnosis and contacts screening, may undergo lack of diagnostic kits. Anyway, as PCR and imaging do not explore the same issues, an integration between clinics, laboratory and imaging appears to be the most effective way of management [8]. This is further true considering that asymptomatic patients may have viral pneumonia features [32] so that a widespread use of imaging may be useful to identify these cases, and CXR with automated image analysis is quick to perform, with limited radiation load, and cost effective.

Finally, from a general Public Health and Big Data in Health perspective, accumulation of CXR images and patients data in large databases, possibly shared at global level, may give the possibility of repeating more in-deep analyses, thus offering the possibility of a continuous, progressive refinement of the knowledge, in a framework of "new light through old windows", performing longitudinal, retrospective studies to evaluate the evolution of the disease.

Moreover, it is widely expected that a further pandemic peak could occur during the seasonal influenza virus epidemic [33]. In this scenario it is pivotal to quickly discriminate the pneumonia etiology to effectively play out the pandemic containments' strategies. Lung pathological studies on autoptic tissue from patients with Covid-19 and influenza virus H1N1 pneumonia suggest widespread thrombosis with microangiopathy and vascular angiogenesis are high value discriminators [34]. Texture analysis could improve the CXR image knowledge extraction in this direction.

Several studies limitation should be considered analyzing the reported results. First, images were accessed from a publicly available repository and no additional clinical information was provided. No information related to the temporal interval between the onset of symptoms compared to the CXR acquisition was provided. The number of images of Covid-19 related pneumonia available for the final analysis was relatively low compared to the total number of images (110 out of 219). The non Covid-19 related interstitial pneumonia patients were selected randomly from the entire repository (1345 images). All CXR images were segmented manually, which might not be feasible in the analysis of very large datasets.

However, our high-performance diagnostic results, combined with a robust ensemble machine learning could be considered a proof of concept to test a quick, inexpensive and safe screening system for Covid-19. 
The EML model we propose is a more stable alternative to the single classification models, which are much subject to dataset dimension variations, class imbalances, outliers and so on and so forth [35]. Indeed, the EML model performs well in the two extreme cases of data availability: when data sets are small and when data sets are large and unwieldy [24, 25]. Moreover, the EML provide a score that can be easily evaluated to balance the diagnostic performance (optimizing the sensitivity or the specificity or the global accuracy). Furthermore, to the best of our knowledge, texture analysis diagnostic performance has not been tested on CXR during the Covid-19 pandemic.

Despite the several limitations, this study can lay ground for future researches in this field and help developing more accurate diagnostic tools for this disease.

\section{Declarations}

Competing interests: The authors declare no competing interests.

\section{References}

1. Chen L, Liu W, Zhang Q, et al (2020) RNA based mNGS approach identifies a novel human coronavirus from two individual pneumonia cases in 2019 Wuhan outbreak. Emerg Microbes Infect 9:313-319. https://doi.org/10.1080/22221751.2020.1725399

2. Zhu N, Zhang D, Wang W, et al (2020) A novel coronavirus from patients with pneumonia in China, 2019. New England Journal of Medicine

3. Mahase E (2020) Covid-19: WHO declares pandemic because of "alarming levels" of spread, severity, and inaction. BMJ 368:

4. Wang D, Hu B, Hu C, et al (2020) Clinical characteristics of 138 hospitalized patients with 2019 novel coronavirus-infected pneumonia in Wuhan, China. Jama 323:1061-1069

5. Wölfel R, Corman VM, Guggemos W, et al (2020) Virological assessment of hospitalized patients with COVID2019. Nature $1-5$

6. Wang W, Xu Y, Gao R, et al (2020) Detection of SARS-CoV-2 in different types of clinical specimens. Jama 323:1843-1844

7. Sethuraman N, Jeremiah SS, Ryo A (2020) Interpreting diagnostic tests for SARS-CoV-2. Jama

8. Ai T, Yang Z, Hou H, et al (2020) Correlation of Chest CT and RT-PCR Testing in Coronavirus Disease 2019 (COVID-19) in China: A Report of 1014 Cases. Radiology 200642. https://doi.org/10.1148/radiol.2020200642

9. Li Y, Xia L (2020) Coronavirus disease 2019 (COVID-19): role of chest CT in diagnosis and management. American Journal of Roentgenology 1-7

10. Hosseinpour-Niazi S, Bakhshi B, Betru E, et al (2019) Prospective study of total and various types of vegetables and the risk of metabolic syndrome among children and adolescents. World journal of diabetes 10:362

11. Wong HYF, Lam HYS, Fong AH-T, et al (2020) Frequency and distribution of chest radiographic findings in COVID-19 positive patients. Radiology 201160

12. Remuzzi A, Remuzzi G (2020) COVID-19 and Italy: what next? The Lancet

13. Gillies RJ, Kinahan PE, Hricak H (2016) Radiomics: images are more than pictures, they are data. Radiology 278:563-577

14. Varghese BA, Cen SY, Hwang DH, Duddalwar VA (2019) Texture analysis of imaging: what radiologists need to know. American Journal of Roentgenology 212:520-528 
15. Giambelluca D, Cannella R, Vernuccio F, et al (2019) PI-RADS 3 Lesions: Role of Prostate MRI Texture Analysis in the Identification of Prostate Cancer. Current problems in diagnostic radiology

16. Kolossváry M, Kellermayer M, Merkely B, Maurovich-Horvat P (2018) Cardiac computed tomography radiomics. Journal of thoracic imaging 33:26-34

17. Fang M, He B, Li L, et al (2020) CT radiomics can help screen the coronavirus disease 2019 (COVID-19): a preliminary study. Science China Information Sciences 63:

18. Belfiore MP, Urraro F, Grassi R, et al (2020) Artificial intelligence to codify lung CT in Covid-19 patients. La radiologia medica $1-5$

19. Murphy K, Smits H, Knoops AJ, et al (2020) COVID-19 on the Chest Radiograph: A Multi-Reader Evaluation of an Al System. Radiology 201874

20. Oh Y, Park S, Ye JC (2020) Deep learning covid-19 features on cxr using limited training data sets. IEEE Transactions on Medical Imaging

21. Chowdhury DrM, Rahman T, Khandakar A, et al (2020) Can Al help in screening Viral and COVID-19 pneumonia?

22. Szczypiński PM, Strzelecki M, Materka A, Klepaczko A (2009) MaZda-A software package for image texture analysis. Computer Methods and Programs in Biomedicine 94:66-76.

https://doi.org/10.1016/j.cmpb.2008.08.005

23. Collewet G, Strzelecki M, Mariette F (2004) Influence of MRI acquisition protocols and image intensity normalization methods on texture classification. Magn Reson Imaging 22:81-91.

https://doi.org/10.1016/j.mri.2003.09.001

24. Troisi J, Landolfi A, Sarno L, et al (2018) A metabolomics-based approach for non-invasive screening of fetal central nervous system anomalies. Metabolomics 14:77. https://doi.org/10.1007/s11306-018-1370-8

25. Troisi J, Sarno L, Martinelli P, et al (2017) A metabolomics-based approach for non-invasive diagnosis of chromosomal anomalies. Metabolomics 13:140. https://doi.org/10.1007/s11306-017-1274-z

26. Troisi J, Scala G, Campiglia P, et al (2016) Method for the diagnosis of endometrial carcinoma

27. DeLong ER, DeLong DM, Clarke-Pearson DL (1988) Comparing the areas under two or more correlated receiver operating characteristic curves: a nonparametric approach. Biometrics 44:837-845

28. RDevelopment CORE TEAM R (2008) R: A language and environment for statistical computing. R foundation for statistical computing Vienna, Austria

29. Fan L, Liu S (2020) CT and COVID-19: Chinese experience and recommendations concerning detection, staging and follow-up. Eur Radiol 1-3. https://doi.org/10.1007/s00330-020-06898-3

30. Soldati G, Smargiassi A, Inchingolo R, et al (2020) Is There a Role for Lung Ultrasound During the COVID-19 Pandemic? J Ultrasound Med. https://doi.org/10.1002/jum.15284

31. Soldati G, Smargiassi A, Inchingolo R, et al (2020) Proposal for International Standardization of the Use of Lung Ultrasound for Patients With COVID-19: A Simple, Quantitative, Reproducible Method. J Ultrasound Med. https://doi.org/10.1002/jum.15285

32. Bai Y, Yao L, Wei T, et al (2020) Presumed Asymptomatic Carrier Transmission of COVID-19. JAMA 323:14061407. https://doi.org/10.1001/jama.2020.2565

33. Sajadi M, Habibzadeh P, Vintzileos A, et al (2020) Temperature and Latitude Analysis to Predict Potential Spread and Seasonality for COVID-19. SSRN Electronic Journal. https://doi.org/10.2139/ssrn.3550308

34. Ackermann M, Verleden SE, Kuehnel M, et al (2020) Pulmonary Vascular Endothelialitis, Thrombosis, and Angiogenesis in Covid-19. N Engl J Med. https://doi.org/10.1056/NEJMoa2015432 
35. Elrahman SMA, Abraham A (2013) A review of class imbalance problem. Journal of Network and Innovative Computing 1:332-340

\section{Table}

Table 1: Classification models and Ensemble machine learning diagnostic performance. Performance were evaluated by means of the test dataset. SE = standard error.

\begin{tabular}{|c|c|c|c|c|c|c|c|}
\hline $\begin{array}{l}\text { Classification } \\
\text { Model }\end{array}$ & $\begin{array}{l}\text { Sensitivity } \\
\text { (Value } \pm S E)\end{array}$ & $\begin{array}{l}\text { Specificity } \\
\text { (Value } \pm S E)\end{array}$ & $\begin{array}{l}\text { Positive } \\
\text { Predictive } \\
\text { Value } \\
\text { (Value } \pm \text { SE) }\end{array}$ & $\begin{array}{l}\text { Negative } \\
\text { Predictive } \\
\text { Value } \\
\text { (Value } \pm S E)\end{array}$ & $\begin{array}{l}\text { Positive } \\
\text { Likelihood } \\
\text { Ratio } \\
\text { (Value } \pm \text { SE) }\end{array}$ & $\begin{array}{l}\text { Negative } \\
\text { Likelihood } \\
\text { Ratio } \\
\text { (Value } \pm S E \text { ) }\end{array}$ & Accuracy \\
\hline Naïve Bayes & $0.90 \pm 0.05$ & $0.84 \pm 0.06$ & $0.85 \pm 0.06$ & $0.90 \pm 0.05$ & 5.78 & 0.11 & 0.873 \\
\hline $\begin{array}{l}\text { Generalized } \\
\text { Linear Model }\end{array}$ & $0.84 \pm 0.07$ & $0.94 \pm 0.04$ & $0.93 \pm 0.05$ & $0.86 \pm 0.06$ & 13.42 & 0.17 & 0.889 \\
\hline $\begin{array}{l}\text { Deep } \\
\text { Learning }\end{array}$ & $1.00 \pm 0.00$ & $0.61 \pm 0.09$ & $0.73 \pm 0.07$ & $1.00 \pm 0.00$ & 2.58 & 0.00 & 0.810 \\
\hline $\begin{array}{l}\text { Gradient } \\
\text { Boosted } \\
\text { Trees }\end{array}$ & $1.00 \pm 0.00$ & $0.65 \pm 0.00$ & $0.74 \pm 0.07$ & $1.00 \pm 0.00$ & 2.82 & 0.00 & 0.825 \\
\hline $\begin{array}{l}\text { Artificial } \\
\text { Neural } \\
\text { Network }\end{array}$ & $0.88 \pm 0.05$ & $0.98 \pm 0.02$ & $0.97 \pm 0.03$ & $0.89 \pm 0.05$ & 37.12 & 0.12 & 0.929 \\
\hline $\begin{array}{l}\text { Partial least } \\
\text { squares- } \\
\text { discriminant } \\
\text { analysis }\end{array}$ & $0.67 \pm 0.07$ & $0.93 \pm 0.04$ & $0.91 \pm 0.05$ & $0.74 \pm 0.06$ & 9.44 & 0.35 & 0.800 \\
\hline $\begin{array}{l}\text { Ensemble } \\
\text { Machine } \\
\text { Learning } \\
\text { (cut-off } \\
\text { 132.57) }\end{array}$ & $0.93 \pm 0.04$ & $0.90 \pm 0.05$ & $0.91 \pm 0.04$ & $0.93 \pm 0.04$ & 9.77 & 0.08 & 0.918 \\
\hline $\begin{array}{l}\text { Ensemble } \\
\text { Machine } \\
\text { Learning } \\
\text { (cut-off }= \\
-100.00 \text { ) }\end{array}$ & $1.00 \pm 0.00$ & $0.80 \pm 0.06$ & $0.85 \pm 0.05$ & $1.00 \pm 0.00$ & 5.13 & 0.00 & 0.906 \\
\hline
\end{tabular}

\section{Figures}




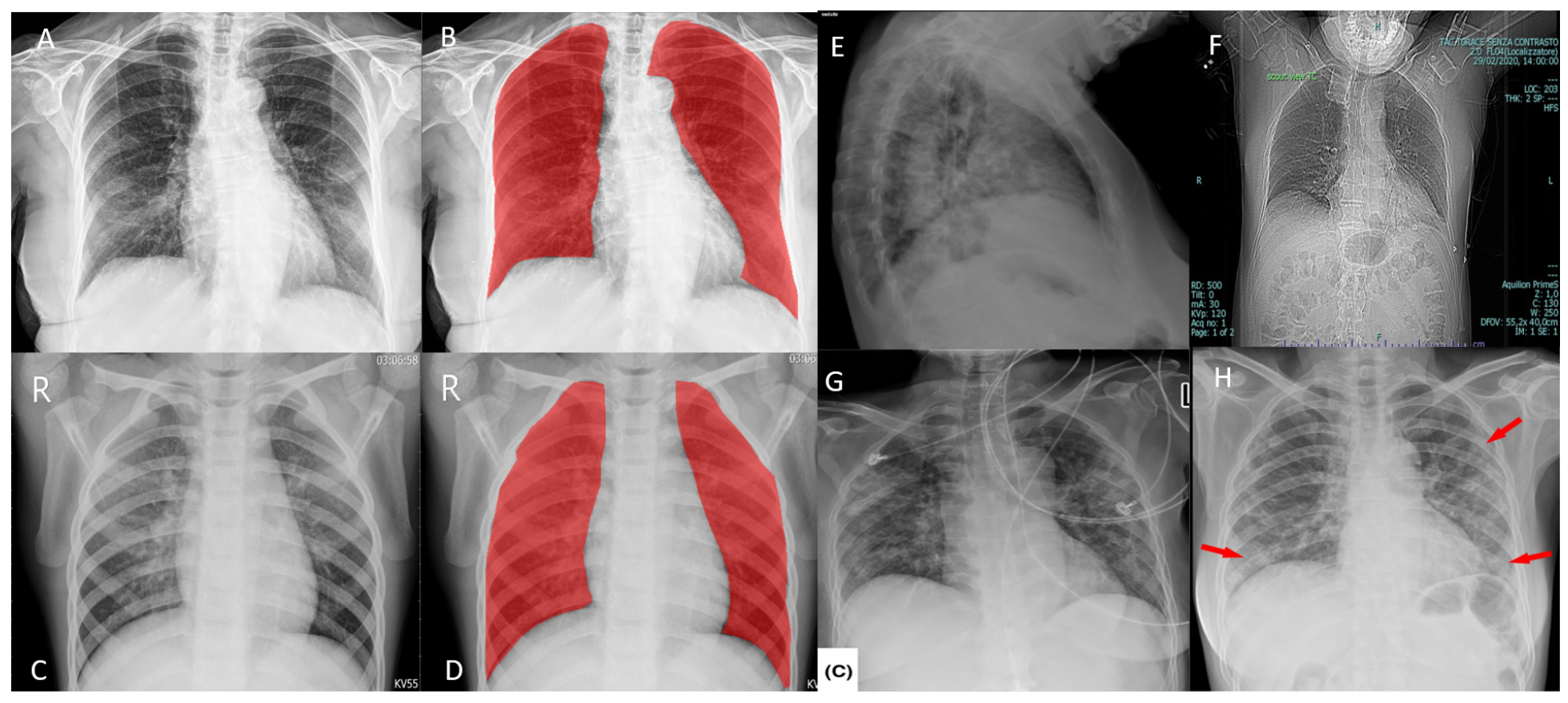

Figure 1

Example of image segmentation of Covid-19 (A-B) and non Covid-19 (C-D) interstitial pneumonia chest x ray using MaZda software. A-B Image \#31, C-D Image \#82. Examples of excluded images of Covid-19 chest $x$ ray. E: LL Projection (\#201). F: Low quality image (\#212). G: ECG wires in the lung areas (\#14). H: annotations in the lung areas (\#62). All images are available at https://www.kaggle.com/tawsifurrahman/covid19-radiography-database.

A

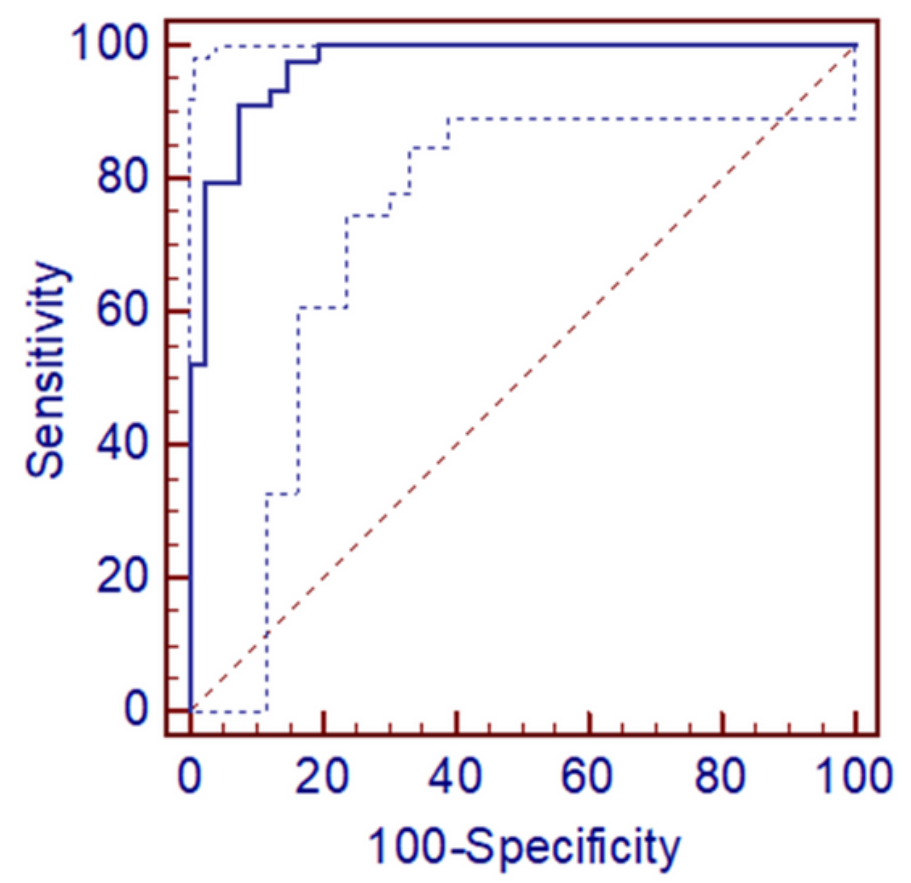

B

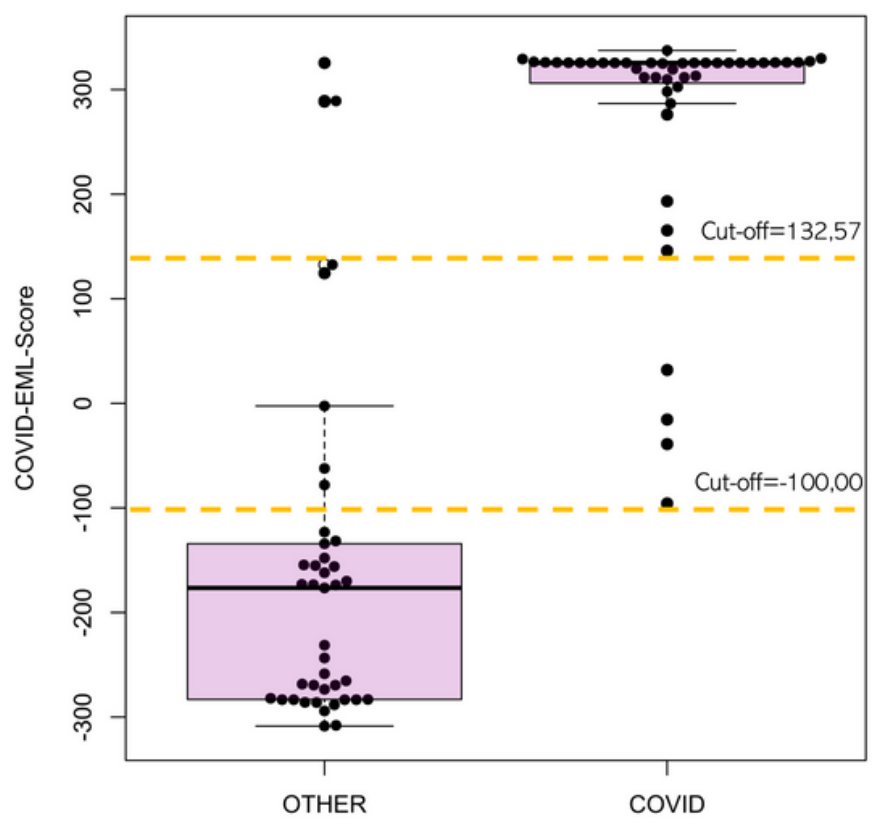

Figure 2

(A) Receiver operating characteristic curve reporting the True Positive Rate (sensitivity) and False Positive Rate (100specificity) for the several investigated Covid-EML-Scores. (B) Box and Whisker plot representation of the Covid-EML- 
Scores for Covid-19 pneumonia X-Ray images and Other pneumonia images. Yellow dashed line indicates the two selected cut-off values (132.57, according to Youden's index and -100.00 according true positive rate optimization).
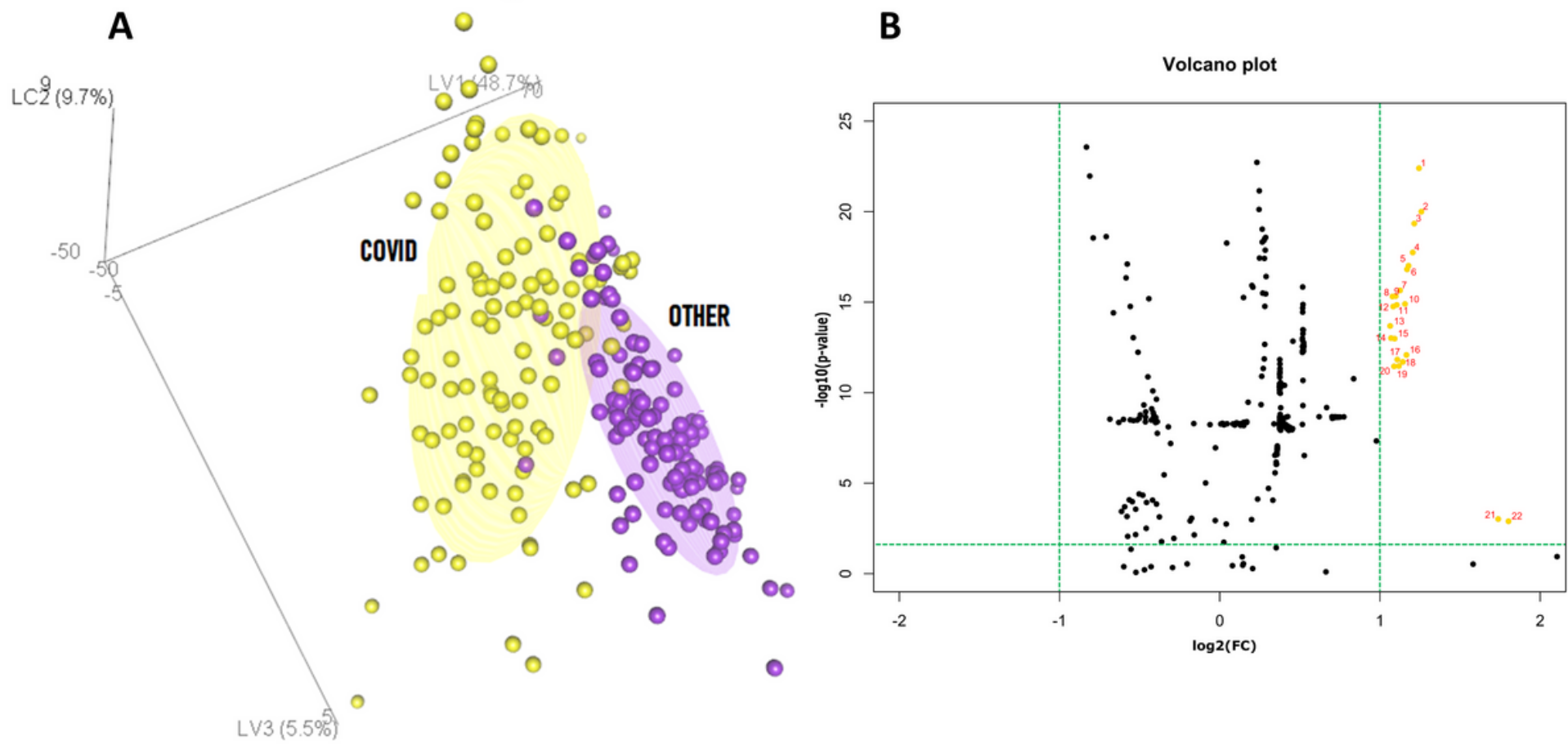

\section{Figure 3}

(A). Graphical representation of the samples' separation achieved by means of Partial Least Square Discriminant Analysis. Axes represent the first 3 latent variables. For each axis the percentage of explained variance were reported in bracket. Ellipses represent 95\% confidence intervals. (B) Volcano plot representation of the investigated features. The ones with an FC>1 and a p-value $<0.05$ are showed in yellow ( $1=45$ dgrLngREmph; $2=135$ drLngREmph; 3=S2_0AngScMom; 4=S1_0AngScMom; 5=S1_1AngScMom; 6=S1_1AngScMom; 7=S3_0AngScMom; 8=S2_2AngScMom; 9=S0_2AngScMom; 10=S2_2AngScMom; 11=S0_3AngScMom; 12=S4_0AngScMom; 13=S0_4AngScMom; 14=S0_5AngScMom; 15=S3_3AngScMom; 16=S5_0AngScMom; 17=S3_3AngScMom; 18=S0_1AngScMom; 19=S4_4AngScMom; 20=S5_5AngScMom; 21=S4_4AngScMom; 22=S5_5AngScMom. 


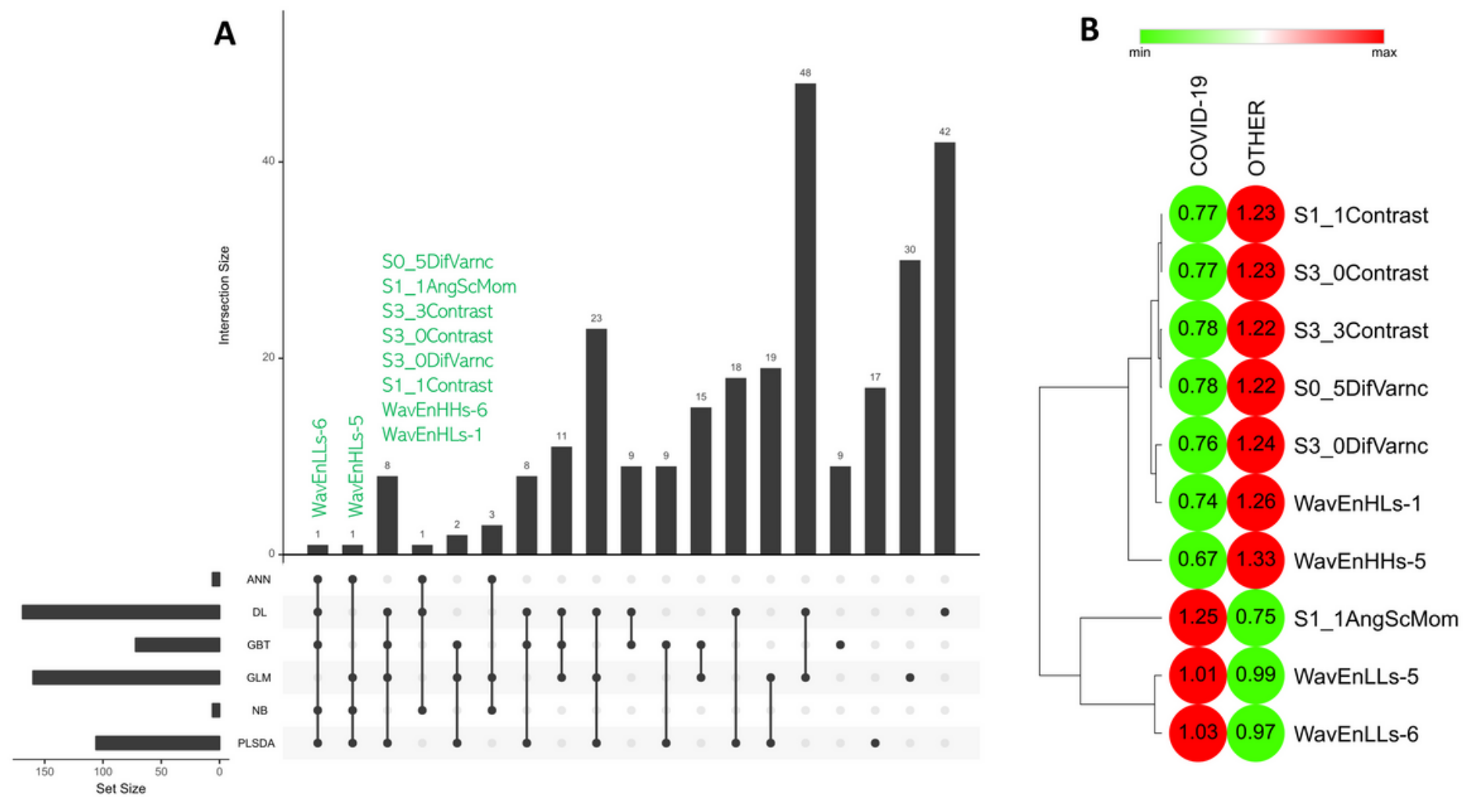

Figure 4

(A) UpSet representation of relevant features (weight $>0$ ) selected from the 6 selected classification models. One feature (WavEnLLs-6) was selected from 5 classification models, while 9 features (in green) were selected from 4 classification models. (B) Heatmap representation of the 10 features selected from at least 4 classification models. Euclidean based clusterization is shown on the right. Green circle indicates a lower mean value in the corresponding class, while red a higher one. Numbers in the circle indicate the normalized mean values.

\section{Supplementary Files}

This is a list of supplementary files associated with this preprint. Click to download.

- SupplementaryS2.xlsx

- SupplementaryS1.html 